\title{
On Exact Solutions of Phi-4 Partial Differential Equation Using the Enhanced Modified Simple Equation Method
}

\author{
Ziad Salem Rached \\ Department of Mathematics, Notre Dame University-Louaize \\ Zouk Mosbeh, Lebanon \\ Email: zrached [AT] ndu.edu.lb
}

\begin{abstract}
Constructing exact solutions of nonlinear ordinary and partial differential equations is an important topic in various disciplines such as Mathematics, Physics, Engineering, Biology, Astronomy, Chemistry,... since many problems and experiments can be modeled using these equations. Various methods are available in the literature to obtain explicit exact solutions. In this correspondence, the enhanced modified simple equation method (EMSEM) is applied to the Phi-4 partial differential equation. New exact solutions are obtained.
\end{abstract}

Keywords--- Nonlinear differential equation, exact solutions, EMSEM, Phi-4 equation

\section{INTRODUCTION}

There is no unique method to solve nonlinear ordinary differential equations (ODE) as well as partial differential equations (PDE). Several techniques were developed and successfully applied by many scientists such as the Expfunction method [1,2], the tanh-function method [3,4], the homogeneous balance method [5,6], the $\left(\mathrm{G}^{\prime} / \mathrm{G}\right)$-expansion method [7,8], the Backlund transformation method [9], the Jacobi elliptic function method [10], the modified simple equation method [11,12], the EMSEM[13].

In the sequel, we obtain exact solutions of Phi-4 PDE

using the EMSEM.

$$
u_{t t}-u_{x x}+m^{2} u+\lambda u^{3}=0
$$

Let us first describe the EMSEM in section II. In Section 3, we apply the method to Phi-4 PDE. Finally, we discuss the results in Section 4.

\section{THE EMSEM}

Suppose we have a nonlinear PDE of the form

$$
F\left(u, u_{t}, u_{x}, u_{x x}, u_{x t}, u_{t t}, \ldots\right)=0
$$

where $F$ is a polynomial of $u$ and its partial derivatives. The method involves four steps.

Step 1: Using the transformation

$$
u(x, t)=u(\zeta), \quad \zeta=p(t) x+q(t)
$$

where $p(t)$ and $q(t)$ are differentiable functions of $t$, from (2) and (3) we get the following ODE

$$
F\left(u,\left(p^{\prime} x+q^{\prime}\right) u^{\prime}, p u^{\prime}, \ldots\right)=0
$$

Step 2: Suppose that equation (4) has the solution 


$$
u(\zeta)=\sum_{k=0}^{n} A_{k}(t)\left[\frac{\varphi^{\prime}(\zeta)}{\varphi(\zeta)}\right]^{k}
$$

where $A_{k}(t)$ are functions of $t, A_{k}(t)$ and $\varphi(\zeta)$ are unknown expressions to be obtained, $A_{n} \neq 0$.

Step 3: Compute $n$ in equation (5). This is accomplished by balancing the highest order derivative and nonlinear term in equation (4).

Step 4: Substitute equation (5) into equation (4). Combine all the terms of the same power of $\varphi(\zeta)^{-j}$, where $j \geq 0$, and equate their coefficients to zero. This results in a system of algebraic and differential equations which can be solved to find $A_{k}(t)$ and $\varphi(\zeta)$. Consequently, we get a closed form solution of equation (2).

\section{APPLICATION TO PHI-4 PDE}

In this section, we solve the Phi-4 PDE

$$
u_{t t}-u_{x x}+m^{2} u+\lambda u^{3}=0
$$

The transformation (3) reduces the above equation to:

$$
u^{\prime}\left(p^{\prime \prime} x+q^{\prime \prime}\right)+u^{\prime \prime}\left(p^{\prime} x+q^{\prime}\right)^{2}-u^{\prime \prime} p^{2}+m^{2} u+\lambda u^{3}=0
$$

Taking the s balance between $u^{\prime \prime}$ and $u^{3}$, we get $n=1$. The solution of equation (6) has the form

$$
u(\zeta)=A_{0}(t)+A_{1}(t)\left[\frac{\varphi^{\prime}(\zeta)}{\varphi(\zeta)}\right]
$$

Our goal is to solve for $A_{0}(t)$ and $A_{1}(t)$. We have

$$
\begin{aligned}
& u^{\prime}=A_{1}(t)\left(\frac{\varphi^{\prime \prime}}{\varphi}-\left(\frac{\varphi^{\prime}}{\varphi}\right)^{2}\right) \\
& u^{\prime \prime}=A_{1}(t)\left(\frac{\varphi^{\prime \prime \prime}}{\varphi}-3 \frac{\varphi^{\prime} \varphi^{\prime \prime}}{\varphi^{2}}+2\left(\frac{\varphi^{\prime}}{\varphi}\right)^{3}\right)
\end{aligned}
$$

Substituting equations (7)-(9) in (6) and setting all the coefficients of $\varphi^{0}, \varphi^{-1}, \varphi^{-1} x, \varphi^{-1} x^{2}, \varphi^{-2}, \varphi^{-2} x, \varphi^{-2} x^{2}$ and $\varphi^{-3}$ to zero, we get respectively

$$
\begin{aligned}
m^{2} A_{0}+\lambda A_{0}^{3} & =0 \\
& q^{\prime \prime} \varphi^{\prime \prime}+\left(q^{\prime 2}-p^{2}\right) \varphi^{\prime \prime \prime}+\left(m^{2}+3 \lambda A_{0}^{2}\right) \varphi^{\prime}=0
\end{aligned}
$$

which, by integration with respect to $\zeta$, reduces to

$$
\begin{aligned}
& q^{\prime \prime} \varphi^{\prime}+\left(q^{\prime 2}-p^{2}\right) \varphi^{\prime \prime}+\left(m^{2}+3 \lambda A_{0}^{2}\right) \varphi=0 \\
& p^{\prime \prime} \varphi^{\prime \prime}+2 p^{\prime} q^{\prime} \varphi^{\prime \prime \prime}=0
\end{aligned}
$$

which, by integration with respect to $\zeta$, reduces to

$$
\begin{aligned}
& p^{\prime \prime} \varphi^{\prime}+2 p^{\prime} q^{\prime} \varphi^{\prime \prime}=0 \\
& p^{\prime 2} \varphi^{\prime \prime \prime}=0 \\
& \quad\left(3 \lambda A_{0} A_{1}-q^{\prime \prime}\right) \varphi^{\prime}-3\left(q^{\prime 2}-p^{2}\right) \varphi^{\prime \prime}=0
\end{aligned}
$$


which, by integration with respect to $\zeta$, reduces to

$$
\begin{aligned}
& \left(3 \lambda A_{0} A_{1}-q^{\prime \prime}\right) \varphi-3\left(q^{\prime 2}-p^{2}\right) \varphi^{\prime}=0 \\
& p^{\prime \prime} \varphi^{\prime}-6 p^{\prime} q^{\prime} \varphi^{\prime \prime}=0 \\
& p^{\prime 2} \varphi^{\prime} \varphi^{\prime \prime}=0 \\
& 2\left(q^{\prime 2}-p^{2}\right)+\lambda A_{1}^{2}=0
\end{aligned}
$$

Various solutions could be obtained. From (10), $A_{0}=0, A_{0}= \pm \frac{m}{\sqrt{\lambda}}$. From (13), or (16) it follows that $p(t)=k$.

From (17), $A_{1}= \pm \sqrt{\frac{2\left(p^{2}-q^{2}\right)}{\lambda}}$.

From (11) and (14), after eliminating $\varphi$, we get

$$
\frac{\varphi^{\prime \prime}}{\varphi^{\prime}}=\left(\frac{q^{\prime \prime}}{p^{2}-q^{\prime 2}}-\frac{3\left(m^{2}+3 \lambda A_{0}^{2}\right)}{\left(-q^{\prime \prime}+3 \lambda A_{0} A_{1}\right)}\right)
$$

Integrating equation (18) with respect to $\zeta$ yields

$$
\varphi^{\prime}=b_{1}(t) \exp (r(t) \zeta)
$$

where

$$
r(t)=\frac{q^{\prime \prime}}{p^{2}-q^{\prime 2}}-\frac{3\left(m^{2}+3 \lambda A_{0}^{2}\right)}{\left(-q^{\prime \prime}+3 \lambda A_{0} A_{1}\right)}
$$

Therefore

$$
\varphi=b_{2}(t)+\frac{b_{1}(t)}{r(t)} \exp (r(t) \zeta)
$$

From (7), (19), and (20), it follows that

$$
u(x, t)=A_{0}+A_{1}\left(\frac{b_{1}(t) \exp (r(t) \zeta)}{b_{2}(t)+\frac{b_{1}(t)}{r(t)} \exp (r(t) \zeta)}\right)
$$

\section{CONCLUSION}

The EMSEM is applied successfully to Phi-4 PDE. Clearly, (21) is an additional set of solutions of the Phi-4 PDE to the ones derived in [11] using the modified simple equation method.

\section{REFERENCES}

[1] J. H. He and X. H. Wu, "Exp-function method for nonlinear wave equations," Chaos, Solitons \& Fractals, vol. 30, no. 3, pp. 700-708, 2006.

[2] M. A. Akbar, N. H. M. Ali, "New Solitary and Periodic Solutions of Nonlinear Evolution Equation by Exp-Function Method,” World Appl. Sci. J., 17 (12), pp. 1603-1610, 2012. 
[3] M. A. Abdou, "The extended Tanh method and its applications for solving nonlinear physical models," Applied Mathematics and Computation, vol. 190, no. 1, pp. 988-996, 2007.

[4] E. Fan, "Extended tanh-function method and its applications to nonlinear equations," Physics Letters A, vol. 277, no. 4-5, pp. 212-218, 2000.

[5] M. L. Wang, "Solitary wave solutions for variant Boussinesq equations," Physics Letters A, vol. 199, no. 3-4, pp. 169-172, 1995.

[6] E. M. E. Zayed, H. A. Zedan, and K. A. Gepreel, "On the solitary wave solutions for nonlinear Hirota-Satsuma coupled KdV of equations," Chaos, Solitons \& Fractals, vol. 22, no. 2, pp. 285-303,2004.

[7] M. Wang, X. Li, and J. Zhang, "The (G'/G)-expansion method and travelling wave solutions of nonlinear evolution equations in mathematical physics, " Physics Letters A, vol. 372, no. 4, pp. 417-423, 2008.

[8] E. M. E. Zayed and K. A. Gepreel, “ The (G'/G)-expansion method for finding the traveling wave solutions of nonlinear partial differential equations in mathematical physics," Journal Math. Phys., 50 (2009) 013502-013514.

[9] M. R. Miura, Backlund Transformation, Springer, Berlin, Germany, 1978.

[10] D. Lu and Q. Shi, "New Jacobi elliptic functions solutions for the combined KdV-MKdV equation," International Journal of Nonlinear science, vol. 10, no. 3, pp. 320-325, 2010.

[11] J. Akter, M. A. Akbar, "Exact solutions to the Benny-Luke equation and the Phi-4 equation by using the modified simple equation method", Results in Physics, 5, pp. 125-130, 2015.

[12] E. M. E. Zayed and S. A. H. Ibrahim, "Exact solutions of nonlinear evolution equations in mathematical physics using the modified simple equation method," Chinese Physics Letters, vol. 29, no. 6, Article ID 060201, 2012.

[13] C. Zhang and Z. Zhang, "Application of the enhanced modified simple equation method for Burger-Fisher and modified Volterra equations," Advances in Difference Equations, 2017. 